\title{
Reduction in Calculated Uncertainty of a Noise Map by Improving the Traffic Model Data Through Two Phases
}

\author{
M. Ausejo, M. Recuero, C. Asensio, I. Pavón \\ Center of Applied Acoustics and non-Destructive Evaluation - CAEND (CSIC-UPM), Serrano 144, \\ 28006, Madrid, Spain.mausejo@i2a2.upm.es
}

\begin{abstract}
Summary
The influence of applying European default traffic values to the making of a noise map was evaluated in a typical environment like Palma de Mallorca. To assess these default traffic values, a first model has been created and compared with measured noise levels. Subsequently a second traffic model, improving the input data used for the first one, has been created and validated according to the deviations. Different methodologies were also examined for collecting model input data that would be of higher quality, by analysing the improvement generated in the reduction in the uncertainty of the noise map introduced by the road traffic noise emission.
\end{abstract}

\section{Introduction}

Drawing up a noise map is a complex procedure for which a large amount of very varied data is required but which is not always available. In the course of preparation, many factors come into play: simplifications, approximations and deviations, which contribute to the final uncertainty of the result.

When making noise maps, the analysis of uncertainty is therefore a key point if the results are to be used as a tool for designing noise action plans. However, to date, the only guides available give an approximate range of the possible contribution of uncertainty in accordance with input data quality $[1,2,3,4]$.

The present research quantifies the uncertainty of the source emission of a noise map in two phases and goes on to evaluate the data improvement process. The two phases are:

- First phase: recommended default traffic data and the use of a large number of approximations.

- Second phase: better quality traffic data and the use of few approximations.

It is important to note that, although there is a wide range of data affecting both the result and the uncertainty of a noise map, the present research only takes into account the effect of using two different traffic models, keeping other essential data such as geometrical data inputs, calculation method, software tool, user calculation settings and other data necessary to carry out a noise map simulation, constant. With this approach undertaken, all the other modelling uncertainties have remained static and only the uncertainty due to the road traffic noise source emission has been assessed.

\section{Uncertainty calculation methods}

A brief description of the general methods to calculate the uncertainty and the process used in this research is described in this section.

\subsection{General methods to calculate the uncertainty}

The problem of the spread of uncertainty is called propagation of distributions and there are several techniques to deal with it [5]:

- Use the Guide to the Expression of Uncertainty in Measurement (GUM) [6] by applying the uncertainty propagation law and the characterization of the final variable using a Gaussian distribution or a t-distribution function.

- Mathematical analytical methods to determine the probability density function of the variable $Y$ (variable related to simulation factors).

- Monte Carlo Method (MCM), which comes to an approximation of the probability distribution of the variable $Y$, by means of input variables random values evaluating the results of the model output.

Apart from the techniques proposed by the GUM, there is the fuzzy logic, which defines values in a real closed interval and applies functions with real numbers to its extension with fuzzy numbers [7]. The biggest problem of fuzzy logic as a tool to determine the uncertainty is the computational cost and complexity [8]. 
With regard to the analytical methods they have a difficult implementation when entailing many variables and there is no information about all of them or their probability density function $(p d f)[9,10]$. In the case of noise maps, where there are more than 40 variables affecting the expanded uncertainty they have not been characterized enough to perform an uncertainty analysis. Even on this premise, certain data related to a noise map (geometrical data) could throw an approximation of its contribution to the total uncertainty through analytical methods [2].

Therefore, MCM is the perfect method to quantify the uncertainty related to a noise map based on their input data [11]. Consequently, MCM has been the method used by the GPG to determine the uncertainty ranges associated with each input data based on its quality. However, the MCM provides results approximate to the exact results that might provide an analytical method [12].

The uncertainty ranges associated with the quality of the data in the GPG are not precise at all, and they have been classified into the following groups:

- Less than $0.5 \mathrm{~dB}$.

- Between 0.5 and $1 \mathrm{~dB}$.

- Between 1 and $3 \mathrm{~dB}$.

- Between 3 and $5 \mathrm{~dB}$.

- Greater than $5 \mathrm{~dB}$.

In addition, the real utility of the uncertainty related to a unique input data, without knowing its contribution to the expanded uncertainty of the complete map is scarce. So there is a real need to determine quantitatively the expanded uncertainty of the outcome of a noise map.

\subsection{Measurement uncertainty calculation}

Although there are several methods, guides, approaches and recommendations to determine the uncertainty of the noise level measurements, authors have chosen to use the updated Guide to the Expression on Uncertainty in Measurements $[6,9]$, as it is the policy document for that purpose. As stated on that document and other references $[13,14]$ the uncertainty of measurement consists of category A (variability of noise source, measurement length, number of samples) and category B contributions (resolution, calibration, AC circuits, A-Weighted filters, linearity, attenuator, RMS, environmental Kit and environmental conditions, among others).

$$
X_{M}=f\left(x_{A}, x_{B}\right) .
$$

\subsection{Simulation uncertainty calculation}

The uncertainty propagation model related to the simulation $\left(Y_{S}\right)$ of a noise map [1] usually considers three main sources of uncertainty,

$$
Y_{S}=f\left(y_{S A}, y_{S B}, y_{S C}\right),
$$

where $y_{S A}$ are contributions related to the acoustic calculation method chosen, $y_{S B}$ are contributions related to the calculation engine used and $y_{S C}$ those related to the creation of the acoustic model.

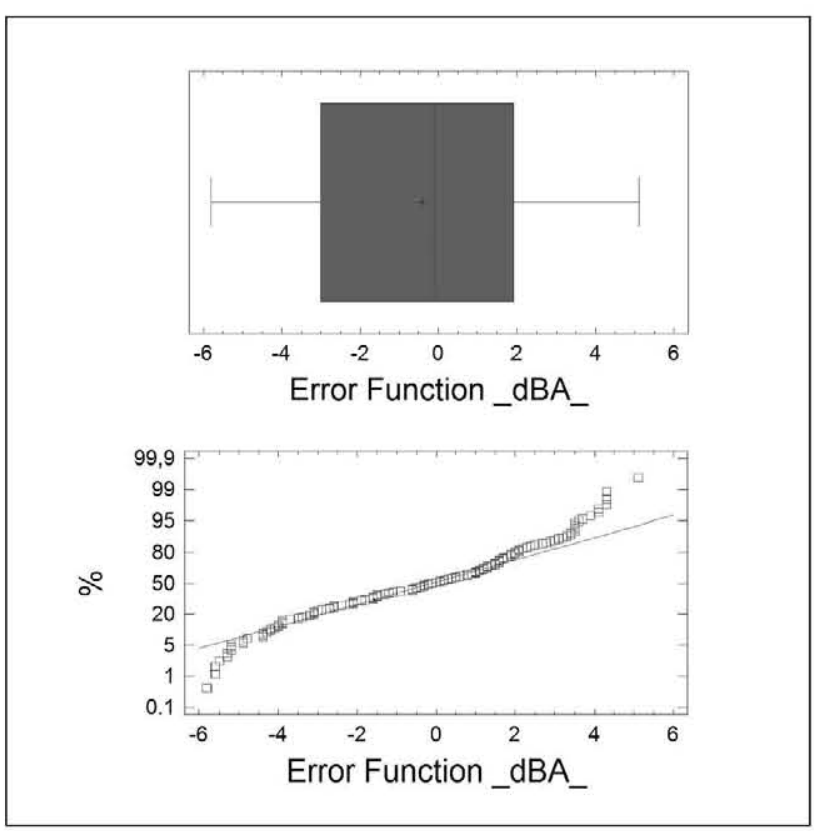

Figure 1. Box-and-whisker diagram (top) and normal probability plot (bottom) for an example of an Error function, with 139 data pairs (measured/simulated values).

Therefore, the total simulation result could be expressed as a function of more than 40 variables $[1,2,15,16]$, producing dependencies among them [4]. Up to date, no analytical study of the probability density functions of all the random variables and their correlation coefficients has been carried out.

\subsection{Uncertainty analysis}

In order to determine the uncertainties in model predictions resulting from uncertainties in the evaluation data, the measured levels and the simulation results have been compared. Thus, to obtain a quantitative value to assess the quality of the input data, a new function has been defined as the Error function $E$,

$$
E=f\left(X_{M}-Y_{S}\right) .
$$

As defined by the revised GUM, the range of values covers the $95.45 \%$ (cover factor $k=2$ ), which implies $\mu \pm 2 \sigma$ (from $\mu-2 \sigma$ to $\mu+2 \sigma$ ) [9]. Therefore, even if the probability density function is not a Gaussian or a symmetrical function (Figure 1), a Confidence Interval (CI) can be determined as a range of values likely to enclose the true value $[6,9]$, which is our proposed method to obtain the Expanded Uncertainty of the simulation model, as shown as an example in Figure 2.

In addition, contributions to the uncertainty of the calculation engine have been reduced, maximizing the simulation time by means of the calculation parameters, without using any time reduction technique (model optimization) and keeping the same user controlled settings, for both Phases 1 and 2 [15]. Thus the uncertainty from the input data and the creation of acoustic model are supposed to charge more weight in the contribution to the total uncertainty. 


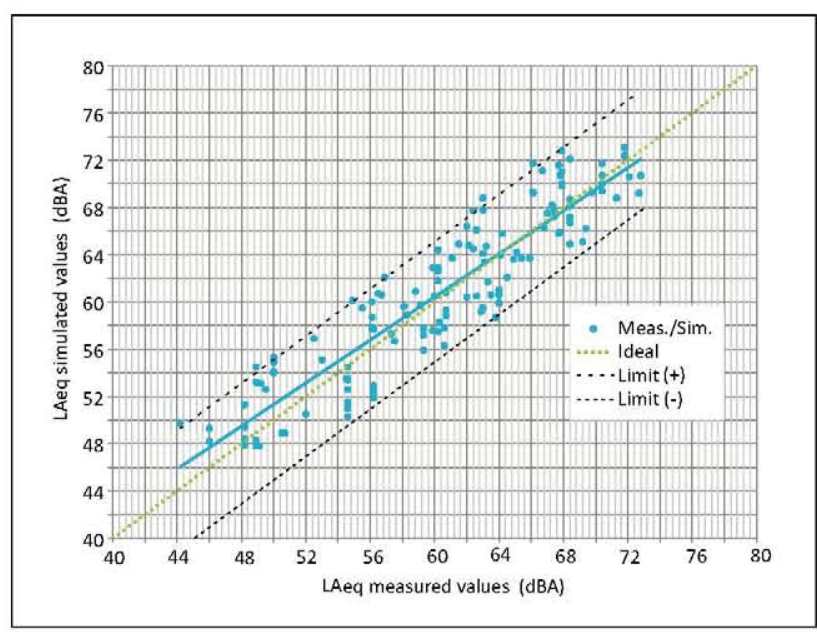

Figure 2. Measured vs. Simulated levels showing the methodological procedure used.

\section{Description of the study area}

The inhabited areas of Palma de Mallorca (Spain) were chosen as the study area, excluding any areas, which, although within the municipal boundaries, were lacking in high population density or which, due to the land use, were not particularly noise-sensitive. This study area was about $200 \mathrm{~km}^{2}$ and formed by close to 400,000 inhabitants. In the study area, road and rail traffic were included as noise sources as there was no industry of note in the area. However, this research will deal exclusively with the uncertainty of road-traffic noise simulation outcomes.

\section{Initial data for preparing the traffic model (Phase 1)}

\subsection{Street layout}

The vast majority of road sections were modelled using a main road with one lane in each direction. This modelling involves a concentration of the linear potential on a single axis, obtaining results similar to those obtained by digitising each lane for large-scale models. As the traffic model available at this Phase 1 only had centre road lanes, it was used taking into account that the traffic flows were homogeneously distributed across the real road lanes [1].

\subsection{Traffic data}

The Department of Mobility supplied traffic data for the year 2006, which was collected using macro-simulation techniques. Supplementary data for the year 2005 was supplied for time distribution by 32 inductive loop detectors at 17 crossroads, distributed throughout the city.

The available data were the Daily Mean Intensity (DMI) for all the days of the whole year, which means that several estimates had to be made to take account of the Day/evening/night distribution annual averages for Light and Heavy vehicles.
In the absence of a large amount of data and the detail required to construct the noise model, a series of measures were taken to obtain the data for the application of default values.

To facilitate traffic data import to Lima v5.1 to create the noise model, a GIS data base was compiled in ArcMap v9.2 so that each road section could be assigned the information needed to characterise the noise emission.

\subsection{Calibrating the traffic model}

As an initial mechanism to control the traffic data, a calibration of the traffic model was performed after noticing considerable differences between the DMI data from the inductive loop detectors and the traffic model.

\subsection{Classifying the roadways}

The streets were then classified so as to be able to use default values and make general assumptions for the whole model in the absence of essential data.

Assigning road types was done according to DMI data from inductive loop detectors, and adjusting it to different recommendations $[1,17,18]$. These recommendations may differ from the real circumstances existing in Palma de Mallorca. However, this general classification appeared to match the circumstances actually examined: "A" (Motorway), "B" (Highway) and "C" (Urban road).

\subsection{Time distribution}

Taking the detailed data from the inductive loop detectors, calculations were made regarding the day/evening/night distribution and mean data were collected that were applied to the entire city. Since they roughly coincided with the recommended default values [1], these values were used $(70 \%, 20 \%$ and $10 \%$ for day, evening and night periods respectively).

\subsection{Traffic adjustment for every day of the year}

Real DMI data were only supplied for working days, for which reason these data were averaged over the 7 days of the week in line with the data collected from the inductive loop detectors in order to get the year-averaged value.

\subsection{Roadways without traffic data}

The traffic model provided only considered the main thoroughfares. The other streets were included in the noise model with DMI default values [1]. Due to the main thoroughfares having been assigned DMI values, values only had to be set for type "C" sections: urban road. However, so that the input data would be consistent, a traffic analysis was undertaken so that there would be no large jumps or discontinuities in the traffic flow.

\subsection{Vehicle speed}

Since no data whatsoever were available for vehicle speed, estimates of effective speed were made according to road type, using the speed limits: $50 \mathrm{~km} / \mathrm{h}$ for the city centre streets and $90 / 120 \mathrm{~km} / \mathrm{h}$ for the ring roads and motorways [1]. 


\subsection{Vehicle acceleration}

Since no data were available concerning acceleration, the sections were designated as "continuous traffic".

\subsection{Light/heavy vehicle distribution}

The distribution of light and heavy vehicles was based on road type, and adapted to internationally recommended criteria [1]. Since no data were available in this respect, the most conservative default values were used, starting out from the hypothesis that these figures would match the established road type (Table I).

Although there are many international classifications for vehicles according to their weight and number of wheels or axles, there are various sets of regulations that simply classify vehicles as light or heavy, although the general trend is to classify vehicles into 5 categories [19]. However, as the END [20] sets the French model, by default, for member nations that do not develop their own calculation model [21], we adopted that model, which categorises vehicles by their weight, as follows: light $(<3500 \mathrm{~kg})$ or heavy $(\geq 3500 \mathrm{~kg})$.

\section{Measurement campaign (Phase 1)}

A measuring campaign was carried out in the months of July and August 2006 at 4 points located at representative roads of each road categorization previously set [22]. Those control points were chosen taken into account the real traffic data available for each road category, after the road categorization establishment [17].

Measurements were taken for 30 days, which means that measurement uncertainty was considerably reduced by having a more than representative sample [23, 24]. All the measurements were carried out under selected meteorological conditions which are reproducible and correspond to quite stable sound propagation conditions, thus during adverse meteorological conditions as rain or high wind speed the measurement data were rejected [25]. These conditions had typical occurrence based on Palma de Mallorca's climate [26].

\section{Validating the road traffic model (Phase 1)}

The effects on the overall error in modelled sound levels, primarily due to the assumptions made during the Phase 1 are being evaluated. Therefore, when measured and simulated values (LAeq for different evaluation periods) were compared (Error function), some very large differences were found, with a maximum deviation of $9.1 \mathrm{~dB}$ and a minimum deviation of $4.0 \mathrm{~dB}$ above the measured values (Figure 3), giving a Confidence Interval of $17.4 \mathrm{~dB}$ (close to a centred $\pm 8.7 \mathrm{~dB}$ Uncertainty) [9].

It is important to note that for the goals pursued in the manuscript, all the noise levels correspond to different simulation data, reason why LAeq can be compared independently of the evaluation period as all the data pairs are
Table I. Default percebtage of heavy vehicles data for road type, suggested by the GPG (Phase 1).

\begin{tabular}{|l|c|c|c|}
\hline Road type & $\begin{array}{c}\text { Day } \\
(07-19 \mathrm{~h})\end{array}$ & $\begin{array}{c}\text { Evening } \\
(19-23 \mathrm{~h})\end{array}$ & $\begin{array}{c}\text { Night } \\
(23-07 \mathrm{~h})\end{array}$ \\
\hline "A" & $20 \%$ & $20 \%$ & $20 \%$ \\
"B" and "C" & $20 \%$ & $15 \%$ & $10 \%$ \\
\hline
\end{tabular}

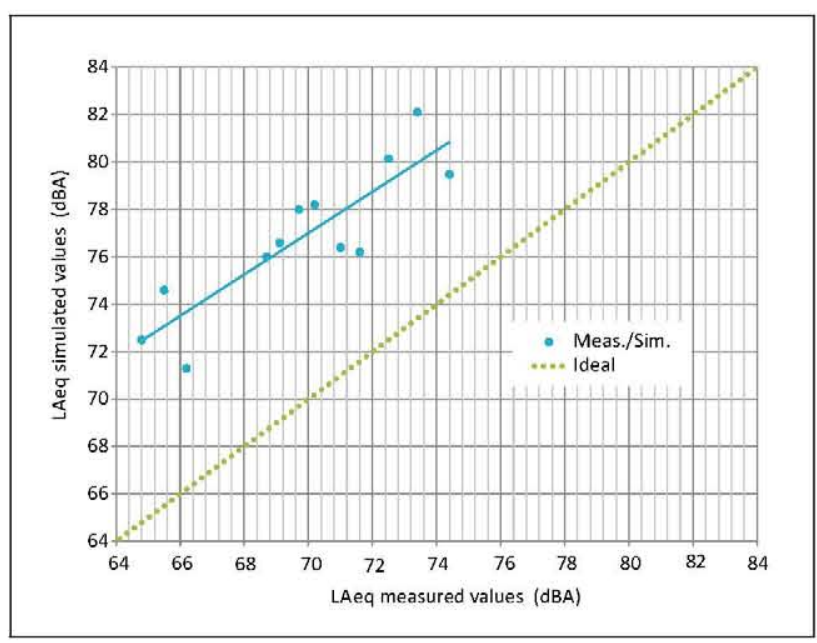

Figure 3. Road traffic model validation (Phase 1).

yearly representative of those conditions. In order to validate comparable data, only measurements during working days were compared to those obtained by means of simulation taking into account the available traffic data.

On observing the regression line of the simulation-measurement data pairs, we could now choose to calibrate the model, subtracting $7.0 \mathrm{~dB}$ from all the linear noise sources [27]. Although by calibrating the simulation model, values better fitted to the ideal line would be obtained and much lower deviations this idea was rejected, since the very high deviations in the model revealed errors and gaps in the simulation model $[28,29]$, thus is not scientifically acceptable.

As the calibration option was rejected, due to the very high deviations, it was decided to make a meticulous analysis of the simulation model and find the most sensitive and least accurate data, with the purpose of performing a new input data collection for the noise model that would produce a noticeable improvement in its quality $[28,30]$.

\section{Improving input data for the road traffic model (Phase 2)}

After examining the errors and gaps in the data entered into the road traffic model, it was found that the least accurate data were the most sensitive: data referring to noise source [1]. It was therefore decided to improve these data.

\subsection{Vehicle intensity and road lanes}

The Department of Mobility provided a new traffic model with data updated to 2007 and consistent with the spot 
measurements of the inductive loop detectors distributed around the city. Duplicated sections and existing errors in the previous traffic model were cleaned up. In addition, the main thoroughfares along the main Avenues were given additional lanes/axis for each direction of flow. Therefore, a new network digitalization was used in the Phase 2 . The total length of the roads with new lanes digitalization was close to $10 \mathrm{~km}$.

\subsection{Classifying roadways}

To supplement the best-quality traffic data, a new road classification was made that was based on Bus Routes, Main Maps, Aerial Photographs, the DMI of the new traffic model and the Department of Mobility's knowledge of the road network.

The classification was as follows:

- "A": Ring Road and Motorways,

- "B": Esplanade and EMT bus routes,

- "C": Major roads,

- "D": Minor roads.

To avoid jumps and discontinuities in noise levels due to errors of classification, the continuity of road categories was carefully checked.

\subsection{Time distribution}

To determine the time distribution of vehicles, data updated to 2007 were available from over 100 inductive loop detectors distributed around the city, representing all the categories previously defined. However, these data did not differentiate between light and heavy vehicles.

The new time distribution obtained after analysing those inductive loop detectors was established to $73 \%, 16 \%$ and $11 \%$ for day, evening and night periods respectively, with slight modifications for every road category.

\subsection{Light and heavy vehicle distribution}

In order to determine the data needed for traffic distribution, various methods were considered. One of the most accurate is the one called Weigh-In-Motion (WIM) which consists in using plaques with piezoelectric components to calculate vehicle weight. However, due to the high cost of systems for calculating vehicle weight, in the absence of the data needed to make the noise map, and the fact that it was one of the most sensitive pieces of data [1, 13], a manual count was undertaken. In order to optimise time, money and accuracy it was decided to view the camera screens of the Department of Mobility on the major thoroughfares, as it was those that carried the highest percentage of heavy vehicles. Six time zones were established that were representative of the time periods being examined (day, evening and night).

Data were obtained analysing the manual counting during 1 hour for a total of 8 locations $\times 6$ time zones each one, and, were therefore very representative of all road categories and all time zones, which proved the time sampling to be adequate.
Table II. New percentage of heavy vehicles data for road type (Phase 2).

\begin{tabular}{|c|c|c|c|}
\hline Road type & $\begin{array}{c}\text { Day } \\
(07-19 \mathrm{~h})\end{array}$ & $\begin{array}{c}\text { Evening } \\
(19-23 \mathrm{~h})\end{array}$ & $\begin{array}{c}\text { Night } \\
(23-07 \mathrm{~h})\end{array}$ \\
\hline "A" & $15 \%$ & $10 \%$ & $5 \%$ \\
"B" & $10 \%$ & $6 \%$ & $3 \%$ \\
"C" & $5 \%$ & $2 \%$ & $1 \%$ \\
"D" & $2 \%$ & $1 \%$ & $0 \%$ \\
\hline
\end{tabular}

Apart from this data, the official data of the number of buses travelling along the city routes were had, making it possible to calculate a very accurate percentage of light and heavy vehicles for every road category (Table II).

\subsection{Vehicle speed data}

Since various studies have made it obvious $[1,13]$ that vehicle speed data have the greatest impact on the quality of the results of the noise map, it was considered essential to improve the quality of the available data in order to improve the final noise model. A method was applied to find this data in an easy, cheap and very accurate way [31]. The method was based on GPS techniques in a floating vehicle for reasons of cost, time and data quality. Measurements were taken for 6 days during the three time periods (day, evening and night) along several roads from all the previously defined noise categories. When speed values had been set for the pre-set noise categories, values were assigned to the roads, creating new noise categories according to speed as follows:

- Motorways: $100 \mathrm{~km} / \mathrm{h}$,

- Ring road: $90 \mathrm{~km} / \mathrm{h}$.,

- Seafront road: $70 \mathrm{~km} / \mathrm{h}$,

- "B" roads: $50 \mathrm{~km} / \mathrm{h}$,

- "C" and "D" roads: $40 \mathrm{~km} / \mathrm{h}$.

\section{Validating the new road traffic model (Phase 2)}

Before the validation of the new model, both Phase 1 and Phase 2 traffic patterns were analysed and any significant change affecting the model was found.

In order to carry out the validation campaign for the new traffic model with the current data, 4 measuring stations were installed for two weeks between June and July 2008. In addition, samples for shorter periods were taken at up to a total of 11 other points, distributed on all the road categories, close to the real traffic data control points using inductive loop detectors.

Analysing the new Error function of Phase 2, the expanded uncertainty for a CI of $95.45 \%$ was $8.2 \mathrm{~dB}$ (close to a centred $\pm 4.1 \mathrm{~dB}$ Uncertainty) (Figure 4).

On examining the new Phase 2 traffic model shown on Figure 4, with the most accurate noise source data, a clear improvement can be seen compared to the old Phase 1 traffic model (Figure 3). 
Table III. Input data quality of each model and its associated uncertainty.

\begin{tabular}{|l|ll|ll|}
\hline & \multicolumn{2}{|c|}{ Initial Model (Phase1) } & \multicolumn{2}{c|}{ Final Model (Phase2) } \\
\hline Input data & Input data quality $\quad$ Uncertainty $(\mathrm{GPG})$ & Input data quality & Uncertainty $(\mathrm{GPG})$ \\
\hline Traffic flow data & Initial data $(2005)$ & - & Final data (2007) & - \\
Road type categorization & 3 categories & - & $4 / 5$ categories & - \\
Traffic flow period distribution & Default values & $1 \mathrm{~dB}$ & Real values & $<0.5 \mathrm{~dB}$ \\
Stretches with no data & Default values & $4 \mathrm{~dB}($ local $)$ & Similar categories approx. & $2 \mathrm{~dB}($ local) \\
Light/Heavy & Default values & $2 \mathrm{~dB}$ & Camera recordings data & $<0.5 \mathrm{~dB}$ \\
Road axis digitalization & 1 single axis & $3-5 \mathrm{~dB}$ & 2 axis (main roads) & $1-3 \mathrm{~dB}($ local) \\
Speed & Speed limit data $\quad 2 \mathrm{~dB}$ & Floating vehicle data & $<0.5 \mathrm{~dB}$ \\
\hline \multirow{2}{*}{$U_{\text {total determined }} 17.4 \mathrm{~dB}(\approx \pm 8.7 \mathrm{~dB})$} & \multicolumn{2}{|c|}{ Un-calibrated: $8.2 \mathrm{~dB}(\approx \pm 4.1 \mathrm{~dB})$} \\
& \multicolumn{2}{|c|}{} & Calibrated: $3.4 \mathrm{~dB}(\approx \pm 1.7 \mathrm{~dB})$ \\
\hline
\end{tabular}

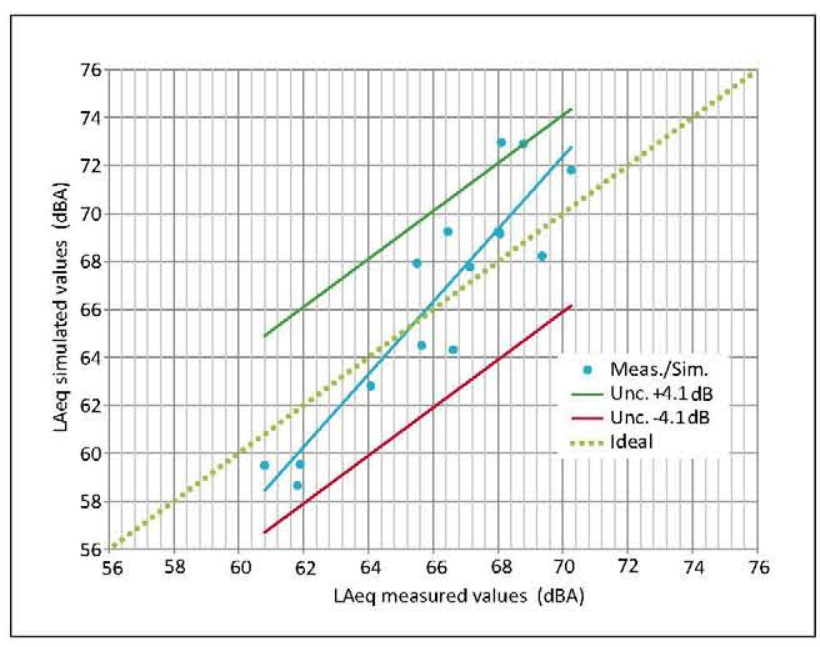

Figure 4. New road traffic model validation (Phase 2).

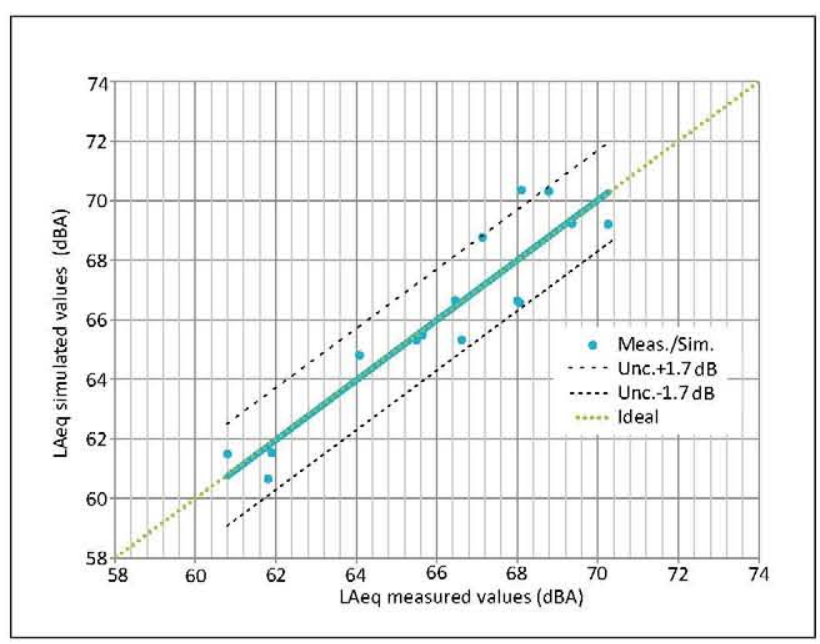

Figure 5. New road traffic model validation (Phase 2) after calibration.

Although the values of uncertainty calculated for the new traffic model were more than acceptable [32], several constant deviations were found between the measured and simulated measurements. These deviations are due to the differences between the acoustic model and the studied area reality (related to traffic data). After analysing them, we realized that there were different systematic deviations for $L_{\text {day }}, L_{\text {evening }}$ and $L_{\text {night }}$. For this reason the model was slightly adjusted (Figure 5), correcting the mean systematic error in each study period (day, evening and night) $[28,30]$, which considerably increased the quality of the final result, obtaining an expanded uncertainty for a CI of $95.45 \%$ of $3.4 \mathrm{~dB}$ (close to a centred $\pm 1.7 \mathrm{~dB}$ Uncertainty).

\section{Analysis of results}

Table III shows a summary of the two models: the approximate uncertainty estimated by the GPG of each piece of input data, [1, 3] and the actual calculated expanded uncertainty. It should be emphasised that the improvement in road classification was not directly quantified. However, it does imply an improvement in accuracy of all the related data.

It should be also highlighted that in the construction of the second noise model none of the input data related to maps, buildings, noise obstacles or propagation conditions was altered, for which reason the influence of the input data on the construction of the noise model is evident. Using the new data collected with the techniques described, and after calibrating the model, mean deviation data of $0.9 \mathrm{~dB}$ was obtained with an expanded uncertainty associated with the model of $3.4 \mathrm{~dB}$. Therefore, the importance attached to collecting noise source-related data is demonstrated [2].

By giving the thoroughfares on the main avenues additional axis, the actual distance to the noise source is reduced. In the specific example of the main avenues, with four lanes in each direction, an improvement of between 0 and $4 \mathrm{~dB}$ was achieved according to the GPG [1].

Another significant improvement in input data is the assigning of traffic data to the roadways without any data assigned. In the initial model, default values were assigned, while in the final model a traffic study was conducted, assigning similar values to those of nearby roads of the same category. However, both the addition of axis as well as the assigning of traffic values to sections with no data produced local improvements. 
The new road classification is more accurate in the assignation of traffic values as well as in its contribution of vehicles and their speed.

There is also an improvement of more than $1 \mathrm{~dB}$ in the use of percentages for light and heavy vehicles that are closer to reality due to the recommended default values no longer being used. The heavy vehicle percentages firstly assumed (default values suggested by GPG) were high for urban roads in this specific study area; fact that can be claimed by comparing Tables I and II.

\section{Conclusions}

In the first phase, in the absence of the data necessary to construct the road traffic model, internationally recommended default values were used [1]. The problem to be approached was how to measure the total uncertainty of the final noise model by using these data [3]. The default values used, always based on a realist but conservative approach, can involve deviations of over $5 \mathrm{~dB}[1,16]$. However, when the quality of the first model was carefully analysed using experimental measurements with their associated expanded uncertainty, it was found that using these default values for some input parameters involved an expanded uncertainty of nearly $17.4 \mathrm{~dB}$. To improve this result, new data were compiled on the source of road traffic noise and new methods were proposed to collect these data [31].

The most important conclusion here is that the improvement of the noise map creation process came solely and exclusively from the use of better traffic noise source data, while the same quality and processing of data related to acoustic barriers and cartography was maintained. Thus, a specific value of the uncertainty reduction mainly due to traffic data input can be calculated avoiding approximations and range values. It is important to note that only the uncertainty in the emission model has been reduced and it is not known how uncertainty across the whole map is affected.

Of special relevance is having found the input data for effective vehicle speed by the technique used and the methodology for finding the contributions of vehicles according to weight, since this is one of the most influential pieces of data [33].

Correcting geometric positioning of individual traffic lanes appears to have a greater impact on successful modelling of noise from Motorways $(0-4 \mathrm{~dB})$ than correctly traffic flow period distribution (less than $1 \mathrm{~dB}$ ).

By using the floating vehicle technique, real data were compiled on the effective speed between journeys with very high accuracy and low cost [31].

\section{References}

[1] WG-AEN: Assessment of exposure to noise. Good practice guide for strategic noise mapping and the production of associated data on noise exposure, version 2. European Commission Working Group, August 2007.

[2] S. Shilton, H. Van Leeuwen, R. Nota: Accuracy implications of using the WG-AEN good practice guide toolkits. Forum Acusticum, Budapest, Hungary, 2005.
[3] Department for Environment, Food and Rural Affairs: Quantified accuracy of GPG toolkits. Research Project NANR 93: WG-AEN's Good Practice Guide and the Implications for Acoustic Accuracy. DGMR V.2004.1300.00. R011.1. UK, May, 2005.

[4] Department for Environment, Food and Rural Affairs: Quantified accuracy of GPG toolkits. Research Project NANR 208: Noise Modelling. DGMR V.2006.1247.00.R4. UK, May, 2007

[5] JCGM 104: Evaluation of measurement data. Supplement 1 to the "Guide to the expression of uncertainty in measurement" and related documents. JCGM, 2009.

[6] International Organization for Standardization: Guide to the expression of uncertainty in measurement. 2nd ed. ISO, Geneva, 1995.

[7] T. De Muer, D. Botteldooren: Uncertainty in noise mapping: comparing a probabilistic and a fuzzy set approach. IFSA 2003, LNAI 2715, 2003, 229-236.

[8] T. De Muer, D. Botteldooren: Methods for quantifying the uncertainty in noise mapping. Symposium: Managing Uncertainty in Noise measurement and prediction, Le Mans, France, 2005.

[9] JCGM 100: GUM 1995 with minor corrections. Evaluation of measurement data. Guide to the expression of uncertainty in measurement. Évaluation des données de mesure. Guide pour l'expression de l'incertitude de mesure. JCGM, 2008 .

[10] R. Kacker, A. Jones: On unse of Bayesian statistics to make the guide to the expression of uncertainty in measurement consistent. Metrologia 40 (2003) 235-248.

[11] JCGM 101: Evaluation of measurement data. Supplement 1 to the "guide to the expression of uncertainty in measurement". Propagation of distributions using a Monte Carlo method. JCGM, 2008.

[12] M. Cox, B. Siebert: The use of a Monte Carlo method for evaluating uncertainty and expanded uncertainty. Metrologia 43 (2006) 178-188.

[13] M. Ausejo, M. Recuero, C. Asensio, I. Pavón, J. M. López: Study of precision, deviations and uncertainty in the design of the strategic noise map of the macrocenter of the city ofBuenos Aires, Argentina. Environmental Modeling and Assessment 15 (2010) 125-135.

[14] M. Ausejo, M. Recuero, C. Asensio, I. Pavón, R. Pagán Study of uncertainty in noise mapping. Internoise 2010 , Lisboa, September 2010.

[15] P. Hepworth, J. Trow, V. Hill: User controlled settings in noise mapping software. The effects on calculation speed and accuracy. Euronoise 2006, Tampere, Finland, 2006

[16] S. Shilton, H. Van Leeuwen, R. Nota: Error propagation analysis of XPS 31-133 and CRTN to help develop a noise mapping data standard. Forum Acusticum, Budapest, Hungary, 2005.

[17] J. Romeu, S. Jiménez, M. Genescà, R. Capdevila: Spatial sampling for night levels estimation in urban environments. Journal of the Acoustical Society of America 120 (2006) IYI-800.

[18] S. Jiménez, M. Genescà, J. Romeu, A. Sánchez: Estimation of night traffic noise levels. Acta Acustica united with Acustica 94 (2008) 563-567.

[19] Swedish National Road And Transport Research Institute: HARMONOISE. Vehicle categories for description of noise source. HAR11TR-030108-VTI04, 2003. 
[20] Directive 2002/49/EC of the European parliament and of the council of 25 june 2002 relating to the assessment and management of environmental noise.

[21] Nouvelle méthode de prevision du bruit. Routes-96. SETRA-CERTU-LCPC-CSTB

[22] J. M. Barrigón, V. Gómez, J. A. Méndez, R. VílchezGómez, J. M. Vaquero, J. Trujillo: A categorization method applied to the study of urban road traffic noise. Journal of the Acoustical Society of America 117 (2005).

[23] D. Kuehner: Long-term leq errors expected and how long to measure (Uncertainty and noise monitoring). Forum Acusticum, Budapest, Hungary, 2005.

[24] J. Sommerhoff, M. Recuero, E. Suárez: Community noise survey of the city of Valdivia, Chile. Applied Acoustics 65 (2004) 643-656.

[25] ISO 1996-2: Acoustics. Description and measurement of environmental noise. Part 2: Acquisition of data pertinent to land use. International Organization For Standardization, Ginebra, 1987.

[26] I. N. D. M. A. E. de Meteorología: Valores climatológicos normales de Palma de Mallorca. http://www.aemet.es/.
[27] D. Manvell, E. Aflalo: Reverse engineering: guidelines and practical issues of combining noise measurements and calculations. Inter Noise, Istambul, Turkey, 2007.

[28] H. Stapelfeldt, D. Manvell: Optimising uncertainty and calculation time. Forum Acusticum, Budapest, Hungary, 2005.

[29] R. Schmidt: Mapping large area's. Balancing between acoustic data economization and result accuracy.

[30] H. Van Leeuwen: Noise maps and action plans. Relations to accuracy, validation and annoyance. Euronoise 2006, Tampere, Finland, 2006.

[31] C. Asensio, J. M. López, R. Pagán, I. Pavón, M. Ausejo: GPS-based speed collection method for road traffic noise. Transportation Research Part D 14, 2009, 360-366.

[32] S. Shilton: Critical input parameters for road and railway noise modelling. Noise mapping according to the 2002/49/EC. Target quality and input requirements, Ispra, Italy, 2009.

[33] D. Skarlatos, T. Zakinthinos: A simplified model for urban noise prediction. Noise Control Engineering Journal 55 (2007) 